\title{
Efficient Approach for Redundant Reader Elimination for Directional Antenna in RFID Networks
}

\author{
Nazish Irfan and Mustapha C.E. Yagoub \\ School of Electrical and Computer Engineering (EECS) \\ University of Ottawa, Ottawa, Canada
}

\author{
Khelifa Hettak \\ Communication Research Center (CRC) \\ 3701 Carling Avenue, Ottawa, Canada
}

\begin{abstract}
Radio Frequency Identification (RFID) systems, due to recent technological advances, have been used for various advantages in industries like production facilities, supply chain management etc. However, this can require a dense deployment of readers to cover the working area. Without optimizing reader's location and number, many of them can be redundant, reducing the efficiency of the whole RFID system. There are many algorithms proposed by researchers to solve redundant reader problem, but all these algorithms are based on omnidirectional reader antenna patterns, which is not practical. In this paper we present an algorithm for redundant reader elimination for directional antenna. It uses a radio propagation model and also accounts for loss due to multipath fading to model communication between a reader and a tag. The efficiency of the proposed approach was demonstrated while preserving the tag coverage.
\end{abstract}

\section{Introduction}

Radio Frequency Identification (RFID) is based on radio communication for tagging and identifying an object. It consists of two blocks namely, RFID transceivers (readers) and RFID transponders (tags). A RFID tag consists of a small integrated circuit for storing information and an antenna for communication. A basic RFID system is based on wireless communication between a reader and a tag. RFID readers can read information stored in no lineof sight RFID tags in their vicinity and communicate it to central database system through wired or wireless interface. Over the last few years, RFID has drawn a great deal of attention and it is now widely believed that RFID can bring revolutionary changes. Some of the major retailers have already invested significantly in RFID and mandated their manufacturers to place tags on cases and pallets, which resulted in mass production of inexpensive RFID tags [1].

In recent years, more efforts have been made to implement RFID-applications in inventory control and logistics management. RFID-based system leads to significant reduction on processing time and labor as inventory in warehouses can be tracked more accurately in a simple, timely and more efficient manner [2]. Applications like inventory detection and automated product receiving in supply chain management require RFID readers to read tags anywhere within a large geographic area. Since the range of reader-to-tag communication is very limited, readers must be deployed in high densities over the entire area [3]. Therefore, the deployment of RFID systems has generated the RFID network planning problem that needs to be solved for large scale deployment. However, RFID network planning is one of the most challenging problems since it has to meet many requirements for efficient operation of RFID systems [4].

This dense deployment of RFID systems in large scale results in unwanted effects. In fact, when multiple readers share the same working environment and communicate over shared wireless channels, a signal from one reader may reach other readers and can cause interference among readers. Hence, the effect of reader interference on the RFID interrogation range should be analyzed before any large scale deployment of readers in a RFID system [5]. Indeed, unnecessary readers in the network may consume power, which can be wasteful. Therefore, finding redundant readers is of great importance for an optimal deployment of a large RFID network.

The problem of redundant reader elimination has been studied extensively in [6-11] to ensure user that an optimal number of readers is used to cover a specified zone. In this paper, we proposed a redundant reader elimination algorithm based on real directional reader antenna pattern. To account for communication between a reader and a tag, this works uses radio propagation model and also accounts for loss due to multipath fading. We have demonstrated that practical measured/simulated data from commercial or in-house antennas can be efficiently utilized to eliminate redundant readers from RFID networks. The redundant reader elimination techniques can be then used for efficient deployment of readers in large warehouse, manufacturing units in production lines and large retail stores.

The remainder of this paper is organized as follows: Section 2 presents a brief survey of related works. Section 3 discuss the radio propagation model used Section 4 details the proposed algorithm. 
Section 5 presents results and discussions and finally Section 6 concludes the proposed work.

\section{Related work}

During the last decade, the RFID collision problem has been extensively covered in literature. It can be categorized as reader-to-reader interference or reader-to-tag interference. Reader-to-reader interference occurs when the interrogation zones of two readers intersect and can interfere with each other. Two readers may also interfere each other even if their interrogation zones do not overlap. This interference is due to the use of wireless radio frequencies for communication. Reader-to-tag interference occurs when more than one reader try to read the same tag simultaneously. In this type of interference, each reader may believe that it is the only reader communicating with the tag while the tag, in fact, is communicating with multiple readers at the same time. The reader collision problem not only results in incorrect operation but also results in reduction of overall read rate of the RFID system [5, 12]. To separate the individual participant signal from one another, many procedures have been developed. Basically, there are four main procedures namely, the Carrier Sense Multiple Access (CSMA), the Frequency Domain Multiple Access (FDMA), the Time Domain Multiple Access (TDMA) and the Code Division Multiple Access (CDMA) [12].

CSMA enables individual data transmission by detecting whether the communication medium is busy. In CSMA, the interrogation zones of two readers do not overlap. However, the signals at particular tag from two readers can interfere each other and make carrier sensing ineffective in RFID network. FDMA relates to techniques in which several transmission channels on various carrier frequencies are simultaneously available to the communication participants. Since RFID tags do not have frequency tuning circuit, tags cannot select particular frequency for communication. It can be achieved by the addition of a frequency tuning circuit, which adds to the cost of the RFID system. TDMA relates to techniques in which the entire available channel capacity is divided among the participants chronologically. In this technique, each reader is allocated different time slot to avoid simultaneous transmissions. In a dynamic RFID system, time slot should be reshuffled adaptively to get better read rate. In case of mobility, reader may come closer and starts interfering. CDMA uses spread spectrum modulation techniques based on pseudo random codes to spread the data over the entire system. To implement CDMA, extra circuitry will be required at the tag which will increase the cost of the tags. Moreover, to assigning codes to all tags at the development site may be complicated.
Therefore, CDMA may not be a cost effective solution.

There are many algorithms in literature, which cover reader collision problem $[12,13]$. Colorwave is a TDMA based distributed algorithm with no guaranteed method of communicating between neighboring nodes. In this technique, each reader monitors the percentage of successful transmissions and also assumes that the readers are able to detect collisions in the RFID system. HiQ is an online algorithm based on Q-learning to solve the reader collision problem. Q-learning is a form of reinforcement learning. This protocol allocates resources to maximize the number of readers communicating at a single time period and it also minimizes the number of collisions among reader's communication. Pulse is a distributed algorithm based on beaconing mechanism. In this protocol, a specific reader while reading a tag periodically broadcasts a beacon on a separate control channel. Any other readers in the network will sense the control channel for beacon before it starts communicating with the tag. If it does not receive any beacon at a given time, it starts transmitting beacon and starts communicating with the tag. This process is expected to achieve fairness among all readers

$\mathrm{DiCa}$ is a distributed and energy efficient anticollision algorithm similar to Pulse. It also has a data channel and a control channel. Each reader contends through the control channel for the use of data channel and the winner reads the tags through the data channel. This algorithm adjusts the control channel range at twice the radius from the first reader to address the hidden and exposed terminal problem. DiCa consumes less dissipated energy than that of CSMA, ALOHA and Pulse. Gentle is a CSMA based protocol that uses RFID multi-channel and beacon messages to mitigate reader collision. In this algorithm, readers can also put tag information in their beacon messages in order to forward the information to their close readers. In Gentle readers, using these two methodologies can avoid reader collision more efficiently and reduce waiting time of getting tag information

Another approach to avoid collision is to reduce the number of redundant readers in the RFID network. In a RFID network, a reader is redundant if all its tags are also covered by at least one of the other readers in the network. Figure 1 shows a typical example of redundant reader in a RFID network. It consists of three readers R1, R2, R3 and five tags $\mathrm{T} 1$ to $\mathrm{T} 5$. Tags $\mathrm{T} 2, \mathrm{~T} 3$ and $\mathrm{T} 4$ covered by R2 are covered by R1 and R3, respectively. Therefore R2 is redundant reader and can be safely removed without violating the full coverage of tags. Eliminating redundant readers from a RFID network has twofold advantages. First, it increases the lifetime of the overall RFID network by saving the 
wasteful power used by redundant readers. Second, it improves the RFID network service quality due to alleviating the interference between readers.

A simple approach to remove redundant reader is by all readers broadcast query message simultaneously to all tags in their interrogation zone. Each tag will reply by signaling its ID. So, if a reader receives no reply from any tag, it may be called as a redundant reader. The reader treated as redundant reader may be because the reader covers no tags in its interrogation zone or tags could not reply due to reader collision.

There are some major drawbacks to the above approach. First, it requires strict time synchronization among readers, which is not practical in most RFID systems. Secondly, by turning all redundant readers, the network coverage may be violated.

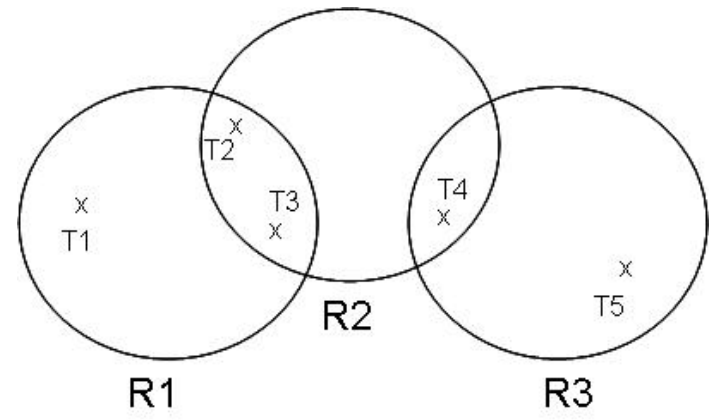

Fig.1 Redundant reader example in RFID network.

The redundant reader elimination (RRE) problem was first introduced by [6]. The RRE algorithm is based on greedy method. The main idea is to record "tag count", i.e., the number of tags a reader covers into RFID tags memory. The reader, which has the maximum tag count, will be the holder of the corresponding tag. In [7], the authors illustrated that RRE procedures fail to eliminate redundant readers for some specific RFID network topologies. Thus, they introduced the LEO algorithm, which uses a layered approach. The fundamental approach of this procedure is "first read first own". In [8], the authors proposed an algorithm, which takes advantage of the concept of neighboring reader density to assess the priority of reading. In this algorithm, the priority value of a reader depends on the number of its neighboring readers. In [9], the authors proposed the algorithm TREE which is very similar to LEO. In [10], the authors introduced the idea that any reader which covers more tags and has fewer number of neighbor readers must be given priority and hence, should be selected. A reader with more neighbors has higher probability of getting its operation interfered by the neighbor readers. In [11] authors propose a greedy algorithm to detect and eliminate redundant readers in RFID networks. The fundamental idea is that reader covering less tags and that has more neighboring readers have higher chance of being redundant.

Redundant reader elimination techniques mentioned above [6-11] are all based on omnidirectional reader antennas, which is not the case for real antennas designed for RFID systems. Moreover, in [14], the authors mentioned that antennas having an omni-directional radiation pattern should be avoided and wherever possible directional antennas should be used because directional antennas have advantages of fewer disturbances to the radiation pattern and to the return loss. Also, omnidirectional antennas have short range and no particular advantage in terms of signal-to-noise ratio (SNR). Therefore, for more realistic applications, redundant reader elimination should be based on directional reader antenna pattern.

In [15], authors have used directional antennas for RFID network planning but did not consider reader antenna tilt for optimization purpose. Authors in [16], used directional antenna, tilt and other parameters for RFID network planning. However, the data of incidence angle between reader and tags are pre-computed for a discrete RFID network; therefore tilt angle for each reader was fixed and not varied. Since tilt angle was not varied for a reader, this approach cannot be used as a generic approach. In this paper we have presented an redundant reader elimination approach, based on directional antenna, which can be effectively utilized with any commercial or in-house directional antenna radiation pattern data.

\section{Radio propagation for RFID deployment and problem definition}

RFID systems are generally operating in indoor environment therefore multipath fading can affect the communication between a reader and a tag [16]. All existing approaches for redundant reader elimination problems [6-11] use distance as a metric to evaluate a reader-to-tag communication. However since, indoor environment can be effected by multipath fading effects, pure distance based metric for simulation will not result in accurate results.

A radio propagation model is required to determine the signal quality received at each tag from the reader. This helps predicting the propagation loss of an electromagnetic field between a reader and a tag in a RFID network. Therefore, in this work we have used propagation model and also considered loss due to multipath to account for the communication between a reader and a tag.

In a RFID system, the passive tag is activated by the reader's forward power which is much more powerful than reverse power sent back by the tag to the reader. Therefore, forward link i.e. reader-to-tag link is the principal issue in the deployment of a RFID network [17]. The simplest way to determine 
the power received by the tag from the reader in free space path loss environment can be given by the Friis equation [18].

$$
P_{r}=P_{t} G_{t} G_{r}\left(\frac{\lambda}{4 \pi d}\right)^{2}
$$

where, $P_{r}$ is received power by tag, $P_{t}$ is transmitted power from reader, $G_{t}$ is transmitter antenna gain, $G_{r}$ is receiver antenna gain, while $d$ determines the length of the direct path between the transmitter and the receiver antenna. The factor $(\lambda / 4 \pi \mathrm{d})^{2}$ determines the free space path loss with " $\lambda$ " the free space wavelength.

Practical deployment of RFID systems in indoor line-of-sight environment involves the in-building path loss. Thus the computation of the propagation loss involves multi-path fading, thus loss due to reflection, path obstruction, absorption and other attenuation effects introduced by the presence of objects inside the indoor site. In this work, we used the indoor propagation model given by [16].

$$
P_{(\text {reader-tag })}=P_{(\text {reader })}-L(d B)
$$

where $P_{(\text {reader })}$ is output power of reader and $L(d B)$ is given by

$$
L(d B)=10 \log \left[\frac{1}{G_{t} G_{r}}\left(\frac{4 \pi}{\lambda}\right)^{2} d^{n}\right]+\alpha[d B]
$$

where $\mathrm{n}$ is the path loss exponent that depends on surroundings and building types and $\alpha$ is $10 \mathrm{~dB}$ in worst case.

Similarly, for reverse link i.e. reflected power from tags to reader, we have [17].

$$
P_{\text {(tag-reader })}=P_{t} T_{b} G_{t}^{2} G_{r}^{2}\left(\frac{\lambda}{4 \pi d}\right)^{2}
$$

where $\mathrm{T}_{\mathrm{b}}$ is the backscatter transmission loss normally taken as $-5 \mathrm{~dB}[17]$.

\section{Proposed algorithm}

In this section we present the proposed technique (RREAD), which has been inspired by [6] and [7], to eliminate redundant readers using real directional reader antennas. Figure 2 shows the typical scenario for redundant reader elimination setup in RFID networks with antenna readers having directional radiation patterns. From this figure, we see that reader 1 can cover tags $1-5$ and similarly reader 2 covers tags $3-5$. Since reader 1 covers all the tags which reader 2 also covers then reader 2 can be eliminated safely without compromising the network coverage.

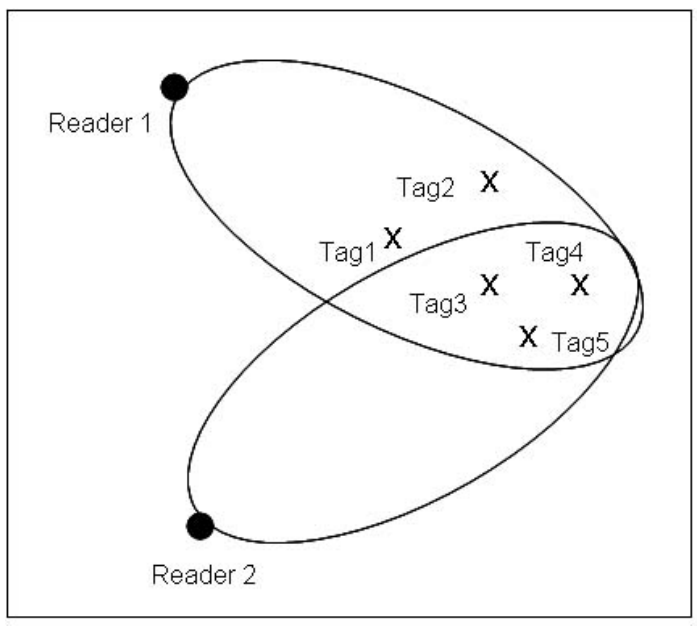

Fig.2 Redundant reader example for directional antenna.

The proposed technique is based on the following assumptions:

1. Reader coordinates are easily available.

2. Coverage information i.e. number of tags each reader has covered in initial round can easily be obtained by data processing.

3. Reader antenna's beam position i.e. tilt angle is known.

It can be noted that the second assumption of collecting coverage information i.e. total number of tags covered by each reader at central host system does not require any new setup to RFID systems. Indeed, since such processing system is already included in existing RFID setup, this assumption adds no extra cost to it. The third assumption is easily available from the installation information of readers.

To test our technique in more realistic situation we have taken six random positions of antenna beam as shown in Figure 3. An antenna coverage is divided into two zones i.e. zone-1 and zone 2 respectively. Each zone is then subdivided into 3 possible positions of a beam. Table 1 shows the different beam positions and respective tilt angles in both zones. Each reader in a network is randomly assigned a beam position out of possible six beam positions. For example suppose we take two readers in a network and assign Reader 1 a beam position 2 and assign Reader 2 a beam position of 6 . With this assignment Reader 1 will have tilt angle of $0^{\circ}$ in zone 1 and Reader 2 will have $-25^{\circ}$ in zone 2 . As the test device, the Intermec RFID reader IA33A circularly polarized panel was adopted and the Intermac UHF tag was used [19].

Now, we will present three different versions of RREAD. 


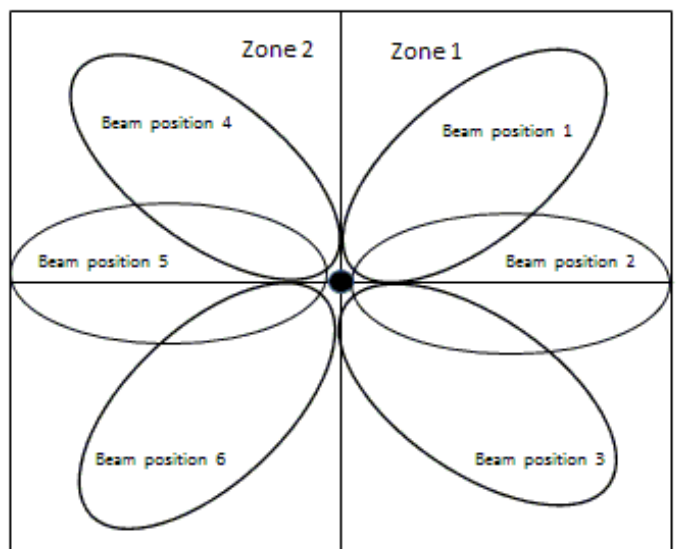

Fig.3 Typical beam position for directional antenna considered for test setup.

Table 1: Tilt angle for each beam positions in different zones

\begin{tabular}{|c|c|c|}
\hline Zone & Beam Position & Tilt Anlge \\
\hline \multirow{3}{*}{ Zone 1 } & 1 & $+25^{\circ}$ \\
& 2 & $0^{\circ}$ \\
& 3 & $-25^{\circ}$ \\
\hline \multirow{3}{*}{ Zone 2 } & 4 & $+25^{\circ}$ \\
& 5 & $0^{\circ}$ \\
\hline
\end{tabular}

RREAD 1 In RREAD 1, the algorithm gives priority to reader which covers the tag first i.e. first read first own. For example in a RFID network suppose there are three readers R1, R2 and R3 and four tags Tag1, Tag2, Tag3 and Tag4. Reader 1 covers Tag1 and Tag2, Reader 2 covers Tag2, Tag3 and Tag 4 and finally Reader 3 covers Tag 3 and Tag4 respectively. By applying RREAD 1, all the tags are covered by Reader 1 and Reader 2 and therefore, Reader 3 is removed as redundant reader. Table 2 summarizes the RREAD 1

Table 2: Simple scenario for RREAD 1

\begin{tabular}{|c|c|c|c|c|}
\hline Reader & Tag1 & Tag2 & Tag3 & Tag4 \\
\hline R1 & R1 & R1 & & \\
\hline R2 & & R2 & R2 & R2 \\
\hline R3 & & & R3 & R3 \\
\hline Final Assignment & R1 & R1 & R2 & R2 \\
\hline Redundant Reader & \multicolumn{5}{|l}{} \\
\hline
\end{tabular}

RREAD 2 In RREAD 2, the algorithm gives priority to reader which covers the maximum number of tags in its coverage zone i.e. the reader which covers the maximum number of tags is given priority. In this technique suppose there are three readers (Reader 1, Reader 2 and Reader 3 ) and four tags (Tag1, Tag2, Tag3 and Tag4). Suppose Reader 1 covers two tags (Tag1 and Tag2), Reader 2 covers three tags (Tag2, Tag3 and Tag4) and Reader 3 covers two tags (Tag3 and Tag4). By applying RREAD 2 Reader 1 holds Tag1, Reader 2 holds
Tag2, Tag3 and Tag4 and finally Reader 3 is eliminated as redundant reader as all the tags in the network are covered by Reader 1 and Reader 2 . Table 3 presents RREAD 2 operation.

Table 3: Simple scenario for RREAD 2

\begin{tabular}{|c|c|c|c|c|}
\hline Reader & Tag1 & Tag2 & Tag3 & Tag4 \\
\hline R1 & (R1:2) & (R1:2) & & \\
\hline R2 & & (R2:3) & (R2:3) & (R2:3) \\
\hline R3 & & & (R3:2) & (R3:2) \\
\hline Final Assignment & R1 & R2 & R2 & R2 \\
\hline Redundant Reader & \multicolumn{5}{|l}{} \\
\hline
\end{tabular}

RREAD 3 RREAD 3 is the combination of RREAD 1 and RREAD 2. In this version of algorithm redundant readers are first removed using RREAD 1 and then use RREAD 2 to eliminate some more redundant readers if possible.

Finally we can summarize the basic operation of our algorithm as:

1. All readers in the RFID network send commands to all the tags in their interrogation zone.

2. Each readers coverage information is sent to the central host station i.e. how many tags (with IDs) each reader has read.

3. Tags are assigned to readers based on RREAD 1, RREAD 2 or RREAD 1+ RREAD 2.

4. Finally all the readers of the network with no tag assigned to them are eliminated as redundant readers.

\section{Simulation setups and results}

\subsection{Simulation setups}

To evaluate the performance of the proposed redundant reader elimination techniques, we implemented three different experimental setups:

1. Setup-1: In this setup five different experimental areas are considered with the numbers of readers and tags are varied in each network.

2. Setup-2: This setup has area of $30 \times 30$ sqm is taken with 75 readers and the numbers of tags are varied from 200 to 400 in five steps.

3. Setup-3: This setup has area of $60 \times 60$ sqm with 500 tags and the numbers of readers are varied from 100 to 200 in five steps.

For each of the experimental areas generated for three setups the location of readers and tags were randomly generated. When random locations for readers and tags in a RFID network have been generated, it was ensured that no reader or tag was located at same position. Table 4 shows the parameters selected for simulations. Figure 4 shows one of the experimental area for setup 1 i.e. the area 
of $30 \times 30$ sq $\mathrm{m}, 75$ readers and 300 tags. Reader gain $7 \mathrm{dBi}$, tag gain $1 \mathrm{dBi}$ and reader power of $30 \mathrm{~dB}$ are taken as simulation parameters for this paper.

Table 4: Parameters of experimental areas (setup 1)

\begin{tabular}{|c|c|c|}
\hline $\begin{array}{c}\text { Working Area } \\
\text { (Square Meter) }\end{array}$ & Number of Readers & Number of Tags \\
\hline $25 \times 25$ & 50 & 200 \\
\hline $30 \times 30$ & 75 & 300 \\
\hline $50 \times 50$ & 100 & 400 \\
\hline $60 \times 60$ & 125 & 500 \\
\hline $75 \times 75$ & 150 & 600 \\
\hline
\end{tabular}

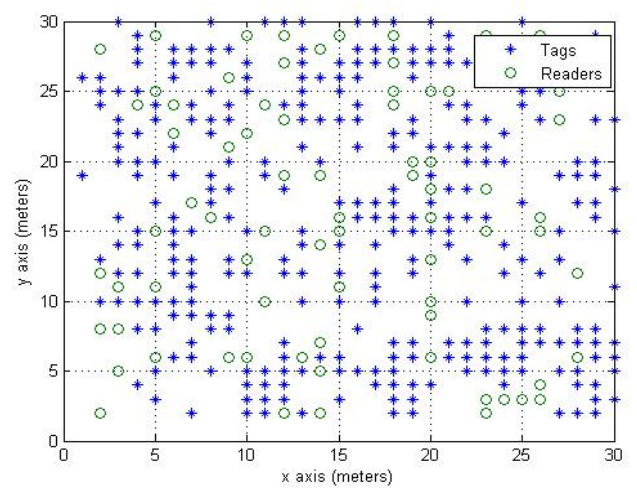

Fig.4 Typical experimental setup.

Since the RREAD is applicable for directional antennas therefore after randomly placing readers and tags in the network each reader is randomly assigned a tilt angle to position the antenna beam as explained in section 4 .

\subsection{Results and discussion}

In this section, we will discuss the performance of our algorithm and analyze the results. Performance evaluation of proposed work was done in two different ways:

1. First, for different experimental areas in setup 1 , coverage attained by readers are evaluated i.e. the total number of tags (in percentage) covered by the remaining readers (after elimination of redundant readers) in the network.

2. Second, for all the experimental areas of setup 1, setup 2 and setup 3, RREAD 1, RREAD 2 and RREAD 3 are implemented and the results analyzed and compared i.e. number of redundant reader eliminated by each technique is evaluated.

It can be seen in Figure 5 that the coverage achieved by readers in different experimental areas of setup 1 range from $94.33 \%--90.33 \%$. This step was undertaken to ensure that appreciable coverage is achieved by readers in the respective experimental areas according to standards used in various simulation setups involving reader elimination [712].

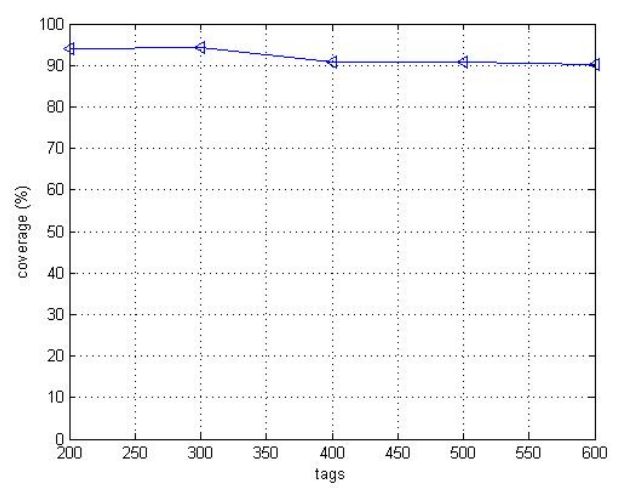

Fig. 5 Coverage achieved by readers in different experimental areas (setup1).

Different versions of RREAD i.e. RREAD 1, RREAD 2 and RREAD 3 are now applied to different experimental areas of setup 1 to evaluate their performances. Figures $6,7,8$ show the number of redundant reader eliminated by using RREAD 1 , RREAD 2 and RREAD 3 respectively for different experimental areas of setup 1 .

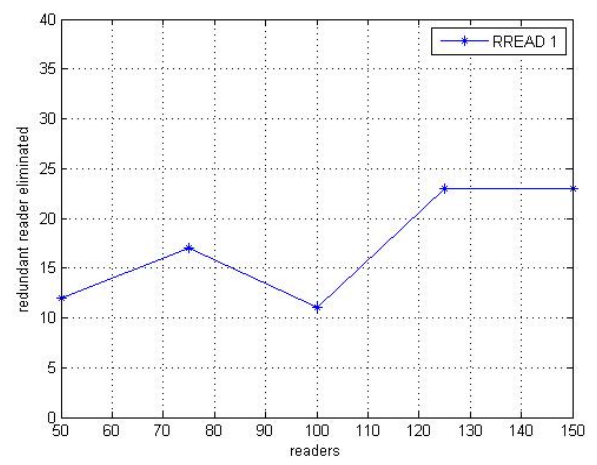

Fig.6 Redundant readers eliminated by RREAD 1 (setup 1).

Figure 9 compares performances of RREAD 1, RREAD 2 and RREAD 3. Similarly, Figure 10 and 11 shows the performance comparison of RREAD 1, RREAD 2 and RREAD 3 for setup 2 and setup 3 respectively.

From Figure 10 it can be easily derived that as the numbers of tags are increased for fixed number of readers in a network the number of redundant readers eliminated is decreased. Similarly, a Figure 11 show that as the numbers of readers are increased for fixed number of tags the number of redundant reader eliminated increases.

It can be observed that for all the test setups RREAD 3 (combination of first read first own and coverage based algorithm) outperforms first read first own algorithm i.e. RREAD 1 and coverage based algorithm i.e. RREAD 2.

The readers are placed randomly in a network and the beam of a directional antenna is narrow. Moreover, for each reader in a network there are not 
many readers with overlapping beams therefore, we can expect that RREAD 3, which is combination of first read first own and coverage based algorithm, to perform better. Performances of algorithms are more dependent on type of RFID network i.e. whether it has sparse or dense deployment of readers in a network.

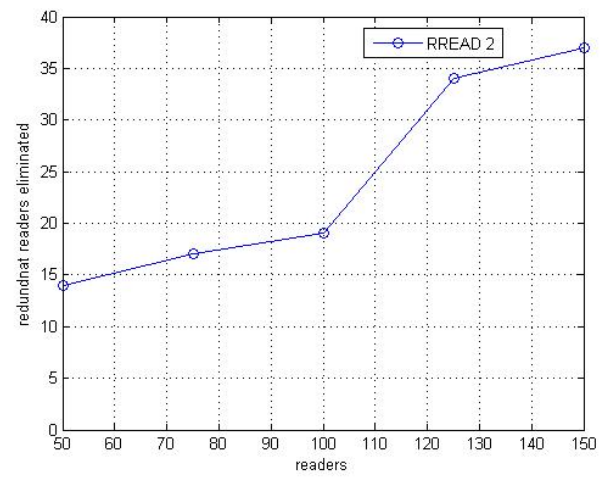

Fig.7 Redundant readers eliminated by RREAD 2 (setup 1).

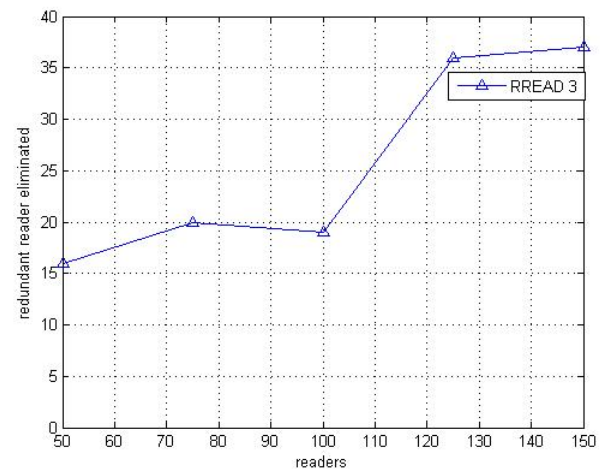

Fig.8 Redundant readers eliminated by RREAD 3 (setup 1).

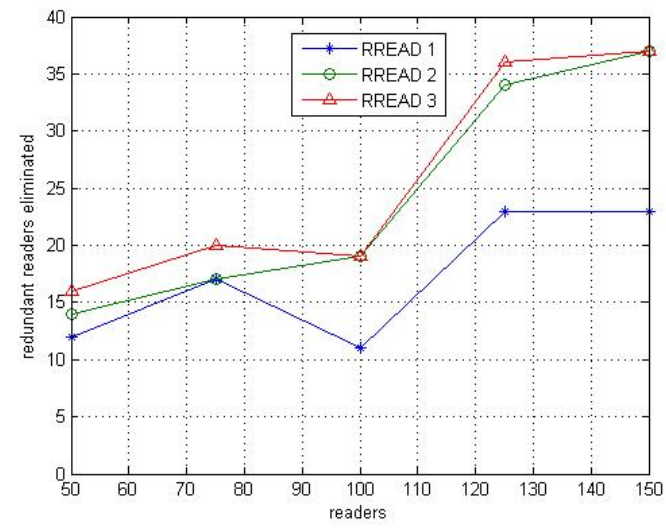

Fig.9 Comparison of redundant readers eliminated using RREAD 1, RREAD 2 and RREAD 3 (setup 1 (setup 1)

Furthermore, most of the redundant reader elimination techniques, based on omni-directional antenna, presented in literature $[6-8,11]$ have many read-write operations. The procedure presented in
[11] has minimum write operations (reader writing or updating information on the tag) whereas the density based procedure [8] has the maximum write operations. Compared to other algorithms, our work has no write operation and has only one read operation. The procedures with more write operations require more resources and eventually add to the cost of operation of RFID systems

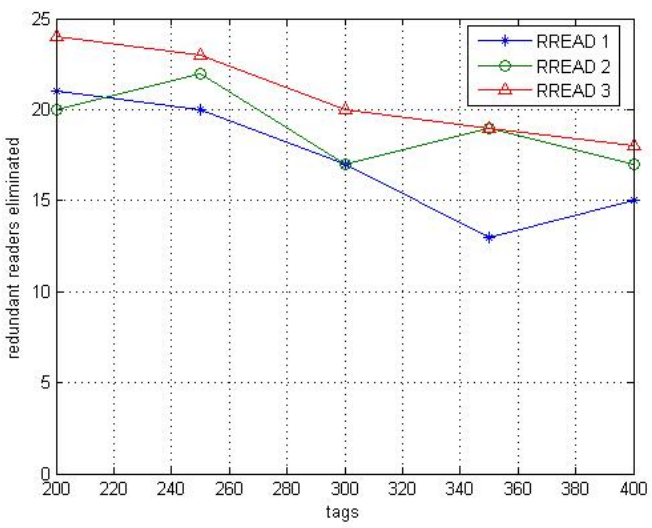

Fig.10 Comparison of redundant readers eliminated using RREAD 1, RREAD 2 and RREAD 3 (setup 2).

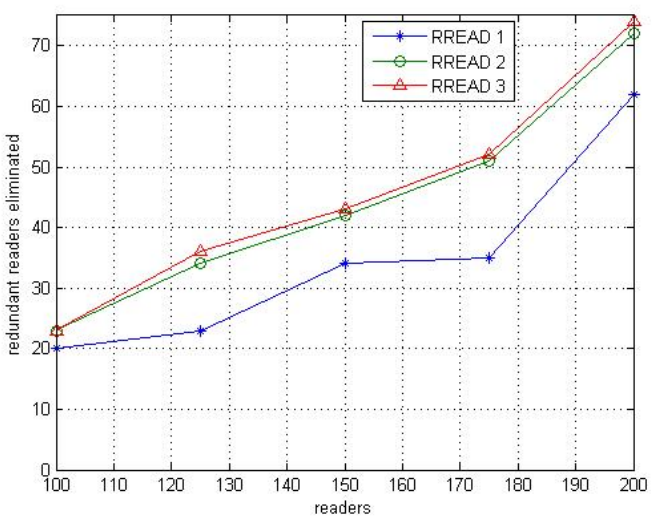

Fig.11 Comparison of redundant readers eliminated using RREAD 1, RREAD 2 and RREAD 3 (setup 3)

Also, since the existing algorithms [6-8,11] require write operations, these procedures are only suitable for tags which have both read and write options. There are mostly three types of tags namely, passive, active and semi-active. Passive tags are lower functionality tags that take power from reader for its operation. Active and semi-active tags have batteries to provide power to tag's operation. Passive Read-Only tags are similar to bar codes in that they are programmed once by a product manufacturer and cannot be altered. Read-Write tags are often called "smart" tags. These tags give users much more flexibility than Read-Only tags. These tags can store large amount of data and have an addressable memory, which can be easily changed. Data on Read-Write tag can be erased and re-written thousands of times. Read-Write operations are only available on tags of EPC Class-2 generation and 
above [20]. However, since tags have extrafunctionality i.e. support read-write operations, increases the cost of RFID system. On the other hand, the proposed work is suitable for any type of tags because it requires no write operation.

\section{Conclusion}

In this paper, we presented a redundant reader elimination technique based on directional antenna radiation pattern. Radio propagation model with loss due to multipath fading is used for reader to tag communication. The proposed work needs only one read and no write operation. This work can be used with any arbitrary RFID network. Simulation results demonstrate that the proposed approach can effectively work as a redundant reader elimination tool for reader with practical directional radiation pattern in RFID network. This work can be used in pre-planning of warehouse and logistics applications to assess the optimal number of RFID readers.

\section{References}

[1] Y. Bendavid, S. F. Wamba, and L. A. Lefebvre, "Proof of Concept of an RFID-Enabled Supply Chain in a B2B eCommerce Environment", in Proceedings of the $8^{\text {th }}$ International Conference on Electronic Commerce (ICEC'06), 2006, pp. 564-568.

[2] Q. Wang, R. McIntosh and M. Antony, "A RFIDbased automated warehouse design", In 2nd International Conference on Computer Engineering and Technology (ICCET), vol. 6, 2010, pp. v6-359 - v6-363.

[3] J. Waldrop, D.W. Engels and S.E. Sarma, "A MAC for RIFD reader networks", In IEEE Wireless Communications and Networking (WCNC), 2003, vol. 3, pp. 1701-1704.

[4] H. Chen and Y. Zhu, "RFID networks planning using evolutionary algorithms and swarm intelligence", In 4th International Conference on Wireless Communications, Networking and Mobile Computing (WiCOM)), 2008, pp. $1-4$.

[5] D. W. Engels, "The Reader Collision Problem", White Paper MIT-AUTOID-WH-007, MIT AUTO ID Center, 2001.

[6] B. Carbunar, M.K. Ramanathan, M. Koyuturk, C. Hoffmann and A. Grama, "Redundant-Reader Elimination in RFID Systems", In Second Annual IEEE Communications and Networks (SECON), 2005, pp. 176184.

[7] C.H. Hsu, Y.M. Chen and C.T. Yang, "A layered optimization approach for redundant reader elimination in wireless RFID networks", In IEEE Asia-Pacific Services Computing Conference, 2007, pp. 138-145.

[8] K.M. Yu, C.W. Yu and Z.Y. Lim, "A density-based algorithm for redundant reader elimination in a RFID network", In Proceedings of the Second International Conference on Future Generation Communication and Networking, 2008, vol. 1, pp. 89-92.

[9] Z.Y. Yang and J.L. Chen, "The simulation and analysis of algorithms for redundant reader elimination in RFID system", Third UKSim European Symposium on Computer Modeling and Simulation, 2009, pp. 494-498.

[10] N. Irfan and M.C.E. Yagoub, "Efficient algorithm for redundant reader elimination in wireless RFID networks", International Journal of Computer Science Issues, 2010, vol. 7(3), pp. 1-8.

[11] K. Ali, W. Alsalish and H.S. Hassanein, "Using neighbor and tag estimations for redundant reader eliminations in RFID networks", IEEE Wirless Communications and Networking Conference (WCNC), pp. 832-837, 2011.

[12] G.P. Joshi and S.W. Kim, "Survey, nomenclature and comparison of reader anti-collision protocols in RFID", IETE Technical Review, 2008, vol. 25, issue 5, pp. 285292.

[13] J. Yu and W. Lee, "GENTLE: Reducing reader collision in mobile RFID networks", in The $4^{\text {th }}$ International Conference on Mobile Ad-hoc and Sensor Networks, 2008, pp. 280-287.

[14] P.R. Foster and R.A. Burberry, "Antenna problems in RFID systems", IEEE Colloquium on RFID Technology, 1999, pp. 3/1-3/5.

[15] Y. Yang, Y. Wu, M. Xia and Z. Qin, "A RFID network planning method based on genetic algorithm", International Conference on Networks Security, Wireless Communications and Trusted Computing, 2009, pp. 534537.

[16] Q. Guan, Y. Liu, Y. Yang and W. Yu, "Genetic approach for network planning in the RFID systems", In 6th International Conference on Intelligent Systems Design and Applications (ISDA), 2006, pp. 567-572.

[17] D.M. Dobkin, "The RF in RFID passive UHF RFID in practice", Elevier Inc., Oxford UK, 2008.

[18] T.S. Rappaport, "Wireless communications principles and practice, second edition", Prentice Hall PTR, New Jersey, 2002.

[19] Intermec, www.intermec.com/products/rfid/antennas. [20] V.D. Hunt, A. Puglia and M. Puglia, "RFID-A guide to radio frequency identification", John Wiley and Sons Inc., Hoboken (N.J.), 2007. 